\title{
Gene transfer for cystic fibrosis
}

\author{
Michael J. Welsh
}

Howard Hughes Medical Institute, University of Iowa College of Medicine, 500 EMRB, Iowa City, Iowa 52242, USA. Phone: (319) 335-7619; Fax: (319) 335-7623;

E-mail: mjwelsh@blue.weeg.uiowa.edu.

Ten years ago the cystic fibrosis (CF) gene was cloned. The discovery of the gene encoding the CF transmembrane conductance regulator (CFTR) $\mathrm{Cl}^{-}$ channel marked the opening of Chapter 1 in the development of gene transfer as a treatment for this common autosomal recessive disease. One year later, Chapter 1 ended when expression of CFTR in CF epithelial cells corrected the $\mathrm{CF} \mathrm{Cl}^{-}$transport defect. From this chapter, we obtained a critical reagent, the CFTR cDNA, and we learned that gene therapy might be feasible.

Chapter 2, the initial experiments in gene transfer to people with CF, began in 1993. Since then several studies have administered the CFTR cDNA to humans using adenovirus, adeno-associated virus (AAV), and cationic lipid vectors. The paper by Harvey et al. in this issue of the JCI (1) brings Chapter 2 to a close. Now it is time to ask what have we learned from this paper and from Chapter 2 , and where to go from here.

Harvey et al (1) sprayed a recombinant adenovirus $\left(\mathrm{E}^{-}, \mathrm{E3}{ }^{-}\right)$encoding CFTR onto a small region of bronchial epithelium in people with CF. By using quantitative RT-PCR, they learned that generation of vector-derived transcripts was dose-dependent and, at the highest doses, the normal CFTR transcripts were present at levels $>5 \%$ of the levels of the endogenous mutated transcripts. $5-10 \%$ is significant because that level might be sufficient to have a clinical

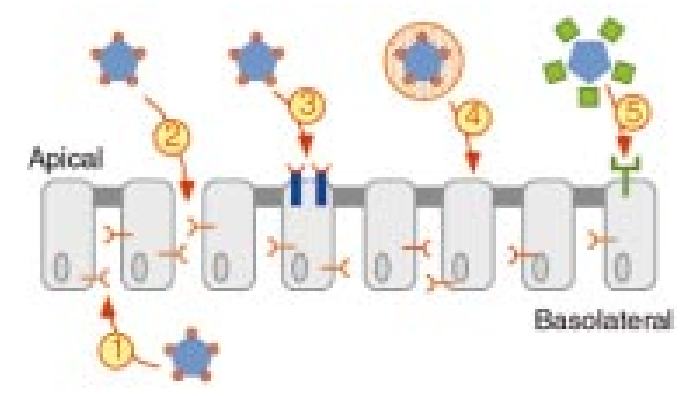

Figure 1

Strategies to enhance gene transfer to airway epithelia. The receptors for currently used viral vectors are shown in red, novel engineered receptors are in blue and red, and potentially useful endogenous apical receptors are in green. The numbered arrows correspond to the strategies listed in the text. The vector is depicted here as a pentagon to emphasize the generality of these approaches. impact, provided all the cells have a few transcripts or transcripts are present in at least $5-10 \%$ of epithelial cells $(2,3)$. Because of technical limitations, the authors could not address these caveats directly, but their data are not inconsistent with these requirements. Thus, the results are encouraging.

In summary, from Chapter 2 we learned that gene transfer to humans with CF works, and gene therapy should work. We now know that CFTR can be expressed in appropriate target tissues, that expression can complement the $\mathrm{CF}$ loss of $\mathrm{Cl}^{-}$transport in vivo in humans, and that gene transfer is safe. Thus, there are no theoretical or fundamental problems that absolutely prevent the development of gene therapy. However, we have also learned of some significant barriers and limitations.

Two challenges that must be met in the next chapter on gene therapy for $\mathrm{CF}$ include limited efficiency of gene transfer and limited persistence of expression. These two issues are related: if the duration of expression could be increased, a lower transduction rate might be acceptable - and, to a lesser extent, the converse.

\section{The challenge of limited efficiency} To obtain $>5 \%$ of transcripts, Harvey et al. applied about $10^{9}$ plaque-forming units ( $\mathrm{pfu}$ ) of vector to a small area. Although the treated area is not given, if one assumes $1.4 \mathrm{~cm}^{2}$, then to obtain a similar result throughout the $1400-\mathrm{cm}^{2}$ surface area from the trachea to the respiratory bronchioles will require $10^{12}$ pfu. This value could increase or decrease depending on the area actually treated. However, with about $3 \times 10^{9}$ potential target cells in the airway, delivery of $10^{12}$ pfu is not very efficient. Several earlier studies also concluded that gene transfer to airway epithelia is quite inefficient. As it turns out, the apical membrane of human airway epithelia provides a significant barrier because it does not express receptors that bind the commonly used adenovirus $(4,5)$, AAV $(6)$, or retrovirus vectors (7). In several cases these receptors are hidden beneath the tight junctions on the basolateral surface where they are not accessible from the airway lumen. Without the advantage of specific high affinity binding and entry into the cell, infection is inefficient.

How can we circumvent this barrier with viral or non-viral vectors? In principle, five general strategies may improve gene transfer efficiency to airway epithelia (Figure 1). 1.) The vector can be delivered from the basolateral surface so that it accesses basolateral receptors. This is easy and works well in vitro, but in vivo it would require blood stream delivery with very specific targeting. 2.) The tight junctions can be transiently disrupted by $\mathrm{Ca}^{2+}$ chelation or hypotonic solutions, allowing vector to penetrate to basolateral receptors (7). 3.) The apical membrane can be modified so that it binds vector. One approach has been to incorporate unnatural sugars into membrane glycoproteins and use them as a molecular handle on which a novel receptor is constructed (8). 4.) New formulation and delivery methods can also enhance binding and gene transfer. Strategies to formulate plasmid DNA into small, uniform complexes are progressing (9, $10)$, and incorporation of viral and nonviral vectors in novel formulations show promise $(11,12)$. 5.) The vectors can be engineered to bear new ligands that bind to the apical surface (13-16). 


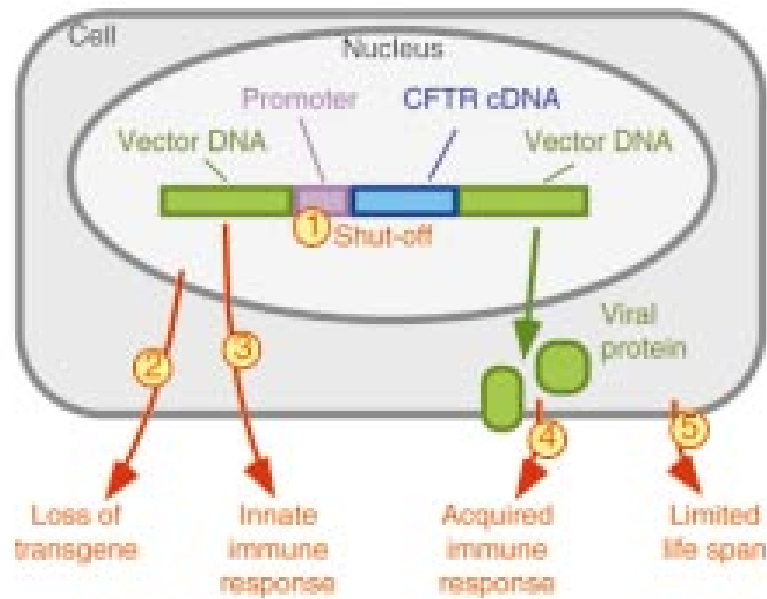

Figure 2

Factors limiting the duration of transgene expression in airway epithelia. The numbered arrows refer to the challenges to persistent expression that are discussed in the text.

Alternatively, new viral vectors or serotypes of existing vectors with improved binding and entry could be identified (17). Independent of binding, limited endocytosis from the apical surface can also pose a problem. The five strategies in Figure 1 apply to most vectors. For non-viral vectors, the additional steps of escape of DNA from endosomes and delivery to the nucleus remain significant hurdles.

\section{The challenge of limited persistence} For a genetic disease like CF, the goal is prolonged expression. In Harvey et al.'s study (1), the expression seen 3 days after vector delivery was not detectable at 30 days. This would necessitate readministration - a requirement complicated by their finding that they could not repeatedly administer the vector and generate measurable transcripts. These results are consistent with several earlier studies. Although Harvey's data suggest that preexisting anti-adenovirus antibodies can reduce expression, their virus application did not itself induce systemic neutralizing antibodies. Presumably, the inability to successfully redose was due to acquired, perhaps cell-mediated, immunity.

Several important factors may limit persistence of CFTR expression (Figure 2). 1.) Promoter "shut-off," first recognized with retroviral constructs, may be overcome using tissue-specific promoters and enhanced viral promoters to achieve sustained expression. 2.) Loss of transgene from the cells is a particular problem for non-viral vectors and for some viral vectors. Integrating viruses and vectors that persist in stable episomal forms may provide solutions. 3.) Another factor that limits transgene persistence arises because $\mathrm{CPG}$ motifs in bacterial DNA contained in plasmid vectors can generate an inflammatory response (18). 4.) Numerous studies have highlighted an immune response that can destroy transduced cells, a problem that is particularly acute when a viral vector retains some viral genes that have leaky transcription or when the transgene expressed is immunogenic $(19,20)$. 5.) Finally, the life-span of differentiated airway cells is limited and is probably in the range of 3 months. Integrating vectors that target progenitor cells could potentially solve this problem.

\section{The chapters ahead}

The progress outlined above is encouraging for developing gene therapy for $\mathrm{CF}$. The principle has been proven and barriers and limitations have been identified. The challenge now is to discover novel strategies to circumvent remaining barriers. Are additional gene transfer experiments in humans useful? Yes, when they test specific hypotheses and help guide future experiments that will take us closer to closing this book.

1. Harvey, B.-G., et al. 1999. Airway CFTR mRNA expression in ctstic fibrosis patients after repetitive administration of recombinant adenovirus. J. Clin. Invest. 104:1245-1255.

2. Chu, C.-S., Trapnell, B.C., Curristin, S.M., Cutting, G.R., and Crystal, R.G. 1992. Extensive posttranscriptional deletion of the coding sequences for part of nucleotide-binding fold 1 in respiratory epithelial mRNA transcripts of the cystic fibrosis transmembrane conductance regulator gene is not associated with the clinical manifestations of cystic fibrosis. J. Clin. Invest. 90:785-790.

3. Johnson, L.G., et al. 1992. Efficiency of gene transfer for restoration of normal airway epithelial function in cystic fibrosis. Nat. Genet. 2:21-25.

4. Pickles, R.J., et al. 1998. Limited entry of adenovirus vectors into well-differentiated airway epithelium is responsible for inefficient gene transfer. J. Virol. 72:6014-6023.

5. Walters, R.W., et al. 1999. Basolateral localization of fiber receptors limits adenovirus infection from the apical surface of airway epithelia. J. Biol. Chem. 274:10219-10226.

6. Summerford, C., and Samulski, R.J. 1998. Membrane-associated heparan sulfate proteoglycan is a receptor for adeno-associated virus type 2 virions. J. Virol 72:1438-1445.

7. Wang, G., et al. 1998. Influence of cell polarity on retrovirus-mediated gene transfer to differentiated human airway epithelia. J. Virol. 72:9818-9826.

8. Lee, J.H., et al. 1999. Engineering novel cell surface receptors for virus-mediated gene therapy. $J$. Biol. Chem. 274:21878-21884.

9. Blessing, T., Remy, J.-S., and Behr, J.-P. 1998 Monomolecular collapse of plasmid DNA into stable virus-like particles. Proc. Natl. Acad. Sci. USA. 95:1427-1431.

10. Perales, J.C., Ferkol, T., Beegen, H., Ratnoff, O.D., and Hanson, R.W. 1994. Gene transfer in vivo: sustained expression and regulation of genes introduced into the liver by receptor-targeted uptake. Proc. Natl. Acad. Sci. USA. 91:4086-4090.

11. Fasbender, A., et al. 1998. Incorporation of adenovirus in calcium phosphate precipitates enhances gene transfer to airway epithelia in vitro and in vivo. J. Clin. Invest. 102:184-193.

12. Worgall, S., Singh, R., Worgall, T., and Crystal, R.G. 1999. Free cholesterol enhances adenoviral vector gene transfer to CAR-deficient cells in vitro. American Society of Gene Therapy, Program of the 2nd Annual Meeting. Washington, DC. June 9-13. p. 131a. (Abstr.)

13. Dmitriev, I., et al. 1998. An adenovirus vector with genetically modified fibers demonstrates expanded tropism via utilization of a coxsackievirus and adenovirus receptor-independent cell entry mechanism. J. Virol. 72:9706-9713.

14. Drapkin, P., Zabner, J., O'Riordan, C., and Welsh, M.J. 1999. Targeting adenovirus binding to the apical membrane of human airway epithelia. American Society of Gene Therapy, Program of the 2 nd Annual Meeting. Washington, DC. June 9-13. p. 190a. (Abstr.)

15. Vigne, E., et al. 1999. RGD inclusion in the hexon monomer provides adenovirus type 5-based vectors with a fiber knob-independent pathway for infection. J. Virol. 73:5156-5161.

16. Wickham, T.J., Roelvink, P.W., Brough, D.E., and Kovesdi, I. 1996. Adenovirus targeted to heparancontaining receptors increases its gene delivery efficiency to multiple cell types. Nat. Biotechnol. 14:1570-1573.

17. Zabner, J., et al. 1999. A chimeric type 2 adenovirus vector with a type 17 fiber enhances gene transfer to human airway epithelia. J. Virol. 73:8689-8695.

18. Krieg, A.M., et al. 1995. CpG motifs in bacterial DNA trigger direct B-cell activation. Nature. 874:546-549.

19. Lieber, A., He, C.Y., Kirollova, I., and Kay, M.A. 1996. Recombinant adenoviruses with large deletions generated by Cre-mediated excision exhibit different biological properties compared with first-generation vectors in vitxro and in vivo. $J$. Virol. 70:8944-8960.

20. Schiedner, G., et al. 1998. Genomic DNA transfer with a high-capacity adenovirus vector results in improved in vivo gene expression and decreased toxicity. Nat. Genet. 18:180-183. 